\title{
THE PHASE SPACE STRUCTURE NEAR NEPTUNE RESONANCES IN THE KUIPER BELT
}

\author{
RENU MALHOTRA \\ Lunar and Planetary Institute, 3600 Bay Area Boulevard, Houston, Texas 77058 \\ Electronic mail: renu@lpis39.jsc.nasa.gov \\ Received 1995 June 20; revised 1995 September 20
}

\section{NASA-CR-204589}

\section{ABSTRACT}

The Solar system beyond Neptune is believed to house a population of small primordial bodies left over from the planet formation process. The region up to heliocentric distance $\sim 50 \mathrm{AU}$ (a.k.a. the Kuiper Belt) may be the source of the observed short-period comets. In this region, the phase space structure near orbital resonances with Neptune is of special interest for the long-term stability of orbits. There is reason to believe that a significant fraction (perhaps most) of the Kuiper Belt objects reside preferentially in these resonance locations. This paper describes the dynamics of small objects near the major orbital resonances with Neptune. Estimates of the widths of stable resonance zones as well as the properties of resonant orbits are obtained from the circular, planar restricted three-body model. Although this model does not contain the full complexity of the long-term orbital dynamics of Kuiper Belt objects subject to the full $\mathrm{N}$-body perturbations of all the planets, it does provide a baseline for the phase space structure and properties of resonant orbits in the trans-Neptunian Solar system. (1) 1996 American Astronomical Society.

\section{INTRODUCTION}

It has long been conjectured, on both theoretical and observational grounds, that the outermost regions of the Solar system may contain a large population of small icy bodies. For example, on the basis of theoretical considerations of the genesis of the planetary system from the primordial Solar Nebula, Kuiper (1951) suggested that a remnant population of icy planetesimals left over from the planet-building process may exist at the current epoch beyond the orbit of Neptune. Whipple (1964) and Bailey (1983) speculated on a massive comet belt as the source of unexplained perturbations of Neptune's orbit [although this argument must now be discarded as the post-Voyager revisions in the planetary ephemeris no longer show any unexplained residuals in Neptune's motion (Standish 1993)]. Hamid et al. (1968) analyzed the orbital plane perturbations of comet $P /$ Halley and concluded that any comet belt between 40 and $50 \mathrm{AU}$ has a total mass less than $1 \mathscr{H}_{\oplus}$. This limit still allows for very large numbers, perhaps $\left(10^{9}\right)$, of cometary bodies.

More recently, it has been suggested that the observed short-period comets with orbital periods $\leqslant 20 \mathrm{yr}$, the "Jupiter family" comets, originate in a belt of low-inclination bodies just beyond the orbit of Neptune, between 35 and $50 \mathrm{AU}$ (Fernandez 1980; Fernandez \& Ip 1983). The older hypothesis that short-period comets originate in a population of near-parabolic Oort Cloud comets which are perturbed into shorter orbits by the giant planets appears unlikely: Duncan et al. (1988), Quinn et al. (1990) have shown that the orbital element distribution of the observed short-period comets is inconsistent with a source in the nearly isotropic Oort Cloud but is compatible with a disk-like source in a trans-Neptune comet belt, which they call the "Kuiper Belt." A possible member of the Kuiper Belt was first discovered in 1992 at a distance of $41 \mathrm{AU}$ from the Sun $\left[1992 \mathrm{QB}_{1}\right.$, reported in Jewitt \& Luu (1993)], and several additional discoveries have been reported since suggesting a potential population of $\sim 35000$ bodies larger than $\sim 100 \mathrm{~km}$ (Jewitt \& Luu 1995).

The dynamical structure of this population has been the subject of several recent theoretical studies (Levison \& Duncan 1993; Holman \& Wisdom 1993; Malhotra 1995). The first two of these (Levison \& Duncan 1993 and Holman \& Wisdom (1993)), studied the long-term stability of test particles in low-eccentricity and low-inclination orbits beyond Neptune, subject only to the gravitational perturbations of the giant planets in their present orbital configuration. They found orbital instability on short time scales $\left(<10^{7} \mathrm{yr}\right)$ interior to 33-34 AU, an intricate structure of interspersed regions of stability and instability in the semimajor-axis range of 34-43 AU, and substantially stable orbits beyond $43 \mathrm{AU}$. The intricacy of the dynamical structure appears to be particularly acute near the locations of orbital resonances with Neptune.

Any primordial trans-Neptune population of planetesimals was undoubtedly subject to dynamical evolution during the planet formation process, and the initial conditions assumed in the above studies are not necessarily representative of the state of the Kuiper Belt at the end of planet formation, as acknowledged in Holman \& Wisdom (1993). The nature of the "dynamical sculpting" of the Kuiper Belt that would have occurred during the late stages of planet formation was the subject of a study by Malhotra (1995). This study concludes that the giant planets' orbits would have evolved significantly and the Kuiper Belt would have been sculpted into a highly nonuniform distribution early in Solar system history: most of the primordial small bodies in the region beyond Neptune's orbit and up to approximately $50 \mathrm{AU}$ heliocentric distance would have been swept into narrow regions of orbital resonances with Neptune, particularly the $3: 2$ and the $2: 1$ orbital resonances which are located at semimajor axes of approximately 39.4 and $47.8 \mathrm{AU}$, respectively. The orbital inclinations of most of these objects would remain 
low (typically $<10^{\circ}$ ), but the eccentricities would be excited to significant values, typically $0.1-0.3$. This structure would be largely preserved to the present epoch.

Pluto (and its satellite Charon) have long been known to reside in the 3:2 Neptune resonance; the resonance libration protects this pair from close encounters with Neptune, despite their Neptune-crossing orbit (with eccentricity $\sim 0.25$ ). Several of the newly discovered Kuiper Belt objects are also likely residents of this resonance (Marsden (1995). (However, note that the orbital parameters of most of these objects remain rather poorly constrained pending future follow-up observations.)

In any case, it is clear that the locations of Neptune's orbital resonances are particularly interesting with regard to the long term dynamics of the Kuiper Belt.

In the region between Neptune's orbit and approximately $50 \mathrm{AU}$ heliocentric distance, by far the most important orbital perturbations are due to Neptune alone, although the $N$-body effects of all the planets, particularly secular resonance effects, also surely play a significant role over the age of the Solar system. In this paper, I use the simplest possible dynamical model, namely, the circular, planar restricted three-body model, to determine the basic phase space structure near the locations of Neptune's orbital resonances exterior to Neptune's orbit. This model does not reflect the complexities that arise with (i) nonzero orbital inclination of the test particle, (ii) a realistic representation of Neptune's motion (its time-varying orbit), and (iii) the perturbations of the other planets. Some of these unmodeled effects can lead to important variations in the dynamics. In particular, as mentioned above, secular resonances in certain regions of phase space can drive the inclinations and eccentricities to large amplitudes and further complicate the dynamics near mean motion resonances. Nevertheless, this model is a reasonable first approximation because (a) the evidence from shortperiod comets suggests a low-inclination source population, (b) in the trans-Neptunian space, there are no first-order orbital resonances with any other giant planets, and (c) Neptune's eccentricity and inclination do not exceed $\sim 0.025$ and $\sim 2.5^{\circ}$, respectively, over billion year time scales under the effects of the mutual gravitational perturbations of the planets. The advantage of this simple model is that it is tractable: it allows for the calculation of two-dimensional surfaces-ofsection in which the phase space structure, in particular, the properties of resonance regions, can be easily visualized. This provides for relatively straightforward estimation of the widths of resonances and the determination of several interesting dynamical properties of resonant orbits, such as the libration periods and their dependence on orbital eccentricity and libration amplitude. To a considerable degree, most of these properties are preserved in the more realistic situation. In short, this model provides a reasonable "baseline" for the phase space structure in the trans-Neptune region.

The rest of this paper is organized as follows. Section 2 describes the technical details of the planar, circular restricted three-body model for the Sun, Neptune, and a Kuiper Belt object, including a description of the information that may be gleaned from surfaces-of-section. Section 3 provides a detailed look at the phase space structure near the major
Neptune resonances of interest for the Kuiper Belt. Section 4 summarizes and discusses the results.

\section{THE PLANAR CIRCULAR RESTRICTED THREE-BODY MODEL}

The classical planar circular restricted three-body problem is a particular case of the general gravitational three-body problem of masses $m_{1}, m_{2}, m_{3}$ defined by the following restrictions:

(1) the motion of all three bodies takes place in a common plane;

(2) the third body, $m_{3}$, has zero mass; therefore it does not influence the motion of $m_{1}$ and $m_{2}$; and

(3) the masses $m_{1}$ and $m_{2}$ describe circular orbits about their common center of mass.

In the context of this paper, $m_{1}$ represents the Sun and $m_{2}$ represents Neptune. The system is made nondimensional by the following choice of units: the unit of mass is taken to be $m_{1}+m_{2}$; the unit of length is chosen to be $a_{N}$ the constant separation between $m_{1}$ and $m_{2}$ (i.e., the mean heliocentric distance of Neptune); the unit of time is chosen such that the orbital period of $m_{1}$ and $m_{2}$ about their center of mass is $2 \pi$. Then the universal constant of gravitation, $G=1$, and the masses of the Sun and Neptune are $1-\mu$ and $\mu$, respectively, where $\mu=m_{2} /\left(m_{1}+m_{2}\right)$. For the Sun-Neptune system, we have $\mu=5.146 \times 10^{-5}$, and this value is used throughout this paper.

In a reference frame with axes $(X, Y)$ rotating with $m_{1}$ and $m_{2}$ and with origin at their center-of-mass, the Sun and Neptune have fixed coordinates, $(-\mu, 0)$ and $(1-\mu, 0)$, respectively, and the third (massless) body has the following equations of motion:

$$
\begin{aligned}
& \ddot{X}=2 \dot{Y}+X-(1-\mu) \frac{X+\mu}{r_{1}^{3}}-\mu \frac{X-1+\mu}{r_{2}^{3}}, \\
& \ddot{Y}=-2 \dot{X}+Y-(1-\mu) \frac{Y}{r_{1}^{3}}-\mu \frac{Y}{r_{2}^{3}},
\end{aligned}
$$

where $r_{1}$ and $r_{2}$ are the test particle's distance to the Sun and to Neptune, respectively,

$$
\begin{aligned}
& r_{1}=\left[(X+\mu)^{2}+Y^{2}\right]^{1 / 2}, \\
& r_{2}=\left[(X-1+\mu)^{2}+Y^{2}\right]^{1 / 2} .
\end{aligned}
$$

These equations of motion admit a constant of the motion, the Jacobi integral, given by

$$
C=X^{2}+Y^{2}-\dot{X}^{2}-\dot{Y}^{2}+2\left(\frac{1-\mu}{r_{1}}+\frac{\mu}{r_{2}}\right) \text {. }
$$

No other constant of the motion is known. For future reference, we note that the Jacobi integral can be expressed in terms of the test particle's semimajor axis, $a$, and eccentricity $e$ :

$$
C=\frac{1}{a}+2 \sqrt{a\left(1-e^{2}\right)}+C(\mu)
$$

The motion of the test particle takes place on a threedimensional subspace (defined by a particular value of $C$ ) embedded in the four-dimensional phase space, $(X, \dot{X}, Y, \dot{Y})$. 

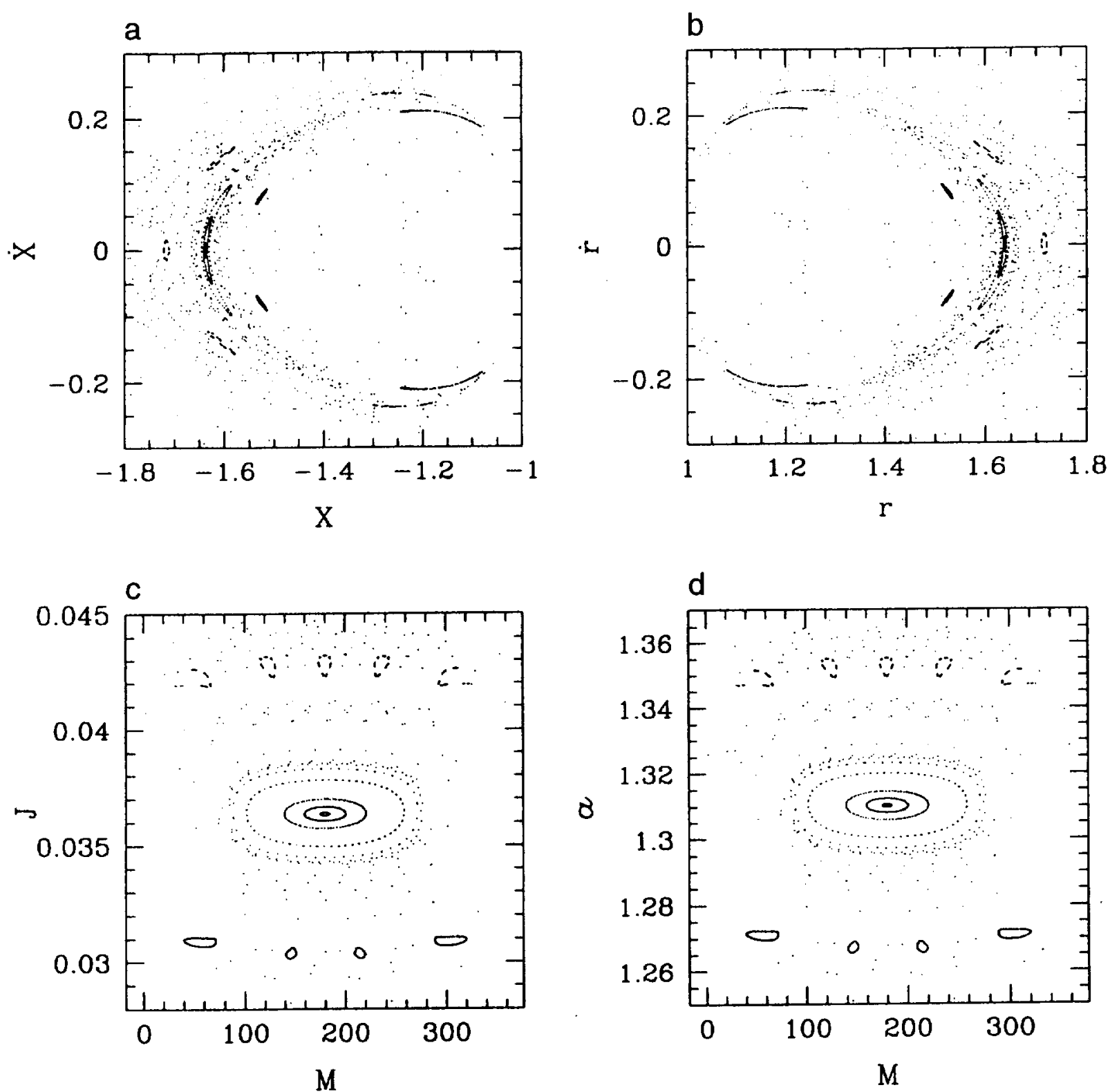

Fig. 1. The phase space structure in the neighborhood of the Neptune-Pluto 3:2 orbital resonance determined from the circular planar restricted three-body model. The same surface-of-section is shown in different variables: (a) cartesian variables, $(X, \dot{X}) ;($ b) plane polar variables, $(r, \dot{r}) ;(c)$ Delaunay variables [mean anomaly $M$, canonical momentum $J=\sqrt{a}\left(1-\sqrt{1-e^{2}}\right)$ ]. (d) is a pseudo-surface-of-section since the semimajor axis $a$ is not a canonical variable. However, qualitatively it is very similar to the surface-of-section (c), and it provides a visualization of the resonance width in terms of the semimajor axis and the amplitude of the perihelion libration.

The usual two-dimensional surface-of-section (s-o-s) is defined by

$$
Y=0, \quad \dot{Y}>0,
$$

and the coordinates on the section are $(X, \dot{X})$. The geometrical interpretation is straightforward: we plot the $X$ coordinate and velocity of the test particle at every conjunction with Neptune. For orbits exterior to Neptune (strictly speaking, with mean motion smaller than Neptune's), this occurs every time the test particle is aligned with the Sun and Neptune and is on the opposite side of the Sun from Neptune; for orbits interior to Neptune (i.e., with mean motion larger than Neptune's), this occurs every time the test particle is aligned with the Sun and Neptune and is on the same side of the Sun as Neptune. For the Solar system beyond Neptune, only the exterior orbits are of interest.

In the s-o-s so defined, periodic orbits of the test particle appear as discrete points. The successive crossings of this surface by a quasiperiodic orbit lie on a closed smooth curve, while a chaotic orbit fills a two-dimensional area.

As the regions in the neighborhood of orbital resonances are of particular interest here, the following remarks are in order.

The location of a $(j+k): j$ orbital period resonance is defined by Kepler's relation between the orbital period and the semimajor axis: 


$$
a=a_{\mathrm{res}}=\left(\frac{j+k}{j}\right)^{2 / 3}
$$

where $j$ and $k$ are integers.

In general, at any resonance, one finds a family of stable periodic orbits that can be parametrized by the orbital eccentricity [or, equivalently, the Jacobi integral, if we use Eq. (6) in Eq. (4)] such that the semimajor axis equals $a_{\text {res }}$ for all orbits in this family, and the eccentricity ranges from zero to some maximum value, $e_{\max }$; for $e>e_{\max }$ the periodic orbit becomes unstable.

In some neighborhood, $\pm \Delta a$ of $a_{\text {res }}$, near each resonance, there exist stable quasiperiodic orbits that librate with finite amplitude about the exact resonant orbit; the half width $\Delta a$ depends upon the mass parameter $\mu$, the resonance itself (i.e., $a_{\text {res }}$ ) and the Jacobi integral $C$.

Let us consider the Neptune-Pluto 3:2 resonance as an illustrative example. If Pluto's inclination is neglected, and if one assumes a fixed circular orbit for Neptune, then the Jacobi integral $C=2.9798$ for the Sun + Neptune+Pluto restricted three-body problem. Figure $1(a)$ is a s-o-s in the neighborhood of the 3:2 Neptune resonance for this value of $C$. This section shows examples of all three types of orbits. The center of the smooth curves lying near $(X, \dot{X})=($ $-1.65,0)$ corresponds to the periodic orbit whose period is exactly $3 / 2$ that of Neptune. The smooth curves are quasiperiodic orbits surrounding this exact periodic orbit and represent test particle orbits that librate about the exact resonance; the size of a smooth curve bears a direct relationship to the amplitude of libration. Such librating orbits are phase protected from having close encounters with Neptune even if the orbit is Neptune crossing. It can be seen that, in general, the regions of quasiperiodic orbits are surrounded by chaotic orbits, i.e., beyond a certain libration amplitude, the smooth curves dissolve into a chaotic zone. Embedded within the "chaotic sea" are other stable zones or chains-of-islands that represent other nearby higher order and secondary resonances. The origin of the chaos is due to the overlap of the higher-order and secondary resonances.

It is perhaps more meaningful to visualize the resonance zone in variables that can be more directly translated into "resonance width." The latter is traditionally (and somewhat loosely) defined as the range in semimajor axis where the orbital perturbations are large. In the restricted three-body problem with $\mu \ll 1$, this is the range in which the amplitude of the orbital parameter variations of the test particle is not simply linearly proportional to $\mu$, but is $\left\langle\left(\mu^{\nu}\right)\right.$ with $\nu \sim 0.5$. In general, in the neighborhood of any resonance, we find regions of stable, librating orbits surrounded by a substantial chaotic zone where test particles may suffer close encounters with Neptune. In this paper, I will define the resonance half width $\Delta a$ as the maximum amplitude of the semimajor-axis variations of stably librating resonant orbits. The value of $\Delta a$ will be estimated from the s-o-s as described below.

The phase space structure seen in the $(X, \dot{X})$ plane can also be seen in other (generalized) canonical coordinates. For example, in plane polar coordinates the equivalent s-o-s $(r, \dot{r}, \theta, \dot{\theta})$ in the inertial reference frame is $(r, \dot{r})$ with the same section condition as in Eq. (5). The s-o-s condition can



FIG. 2. The time variation of the semimajor axis for orbits with $e \approx 0.25$ near the boundary between the stable libration zone and the chaotic zone at the 3:2 Neptune resonance (cf. Fig. 1). (The unit of length is Neptune's semimajor axis, and the unit of time is Neptune's orbital period, approximately $165 \mathrm{yr}$.) The Jacobi integral for all four orbits is $C=2.9798$; the initial conditions $(X, X, Y, \dot{Y})$ for the four orbits are $(\mathrm{a})-(-1.6100,0.0$, $0.0,0.92442), \quad$ (b) $-(-1.6105,0.0,0.0,0.92508), \quad$ (c) $-(-1.6106,0.0,0.0$, $0.92521)$, (d)-(-1.6110,0.0,0.0,0.92574). These orbits are all chaotic and are shown in order of decreasing distance to the stability boundary. The orbits in (a), (b), and (c) become unstable after 7, 31, and $\sim 200$ librations (i.e, $\sim 8 \times 10^{4},-3 \times 10^{5}$, and $\sim 2 \times 10^{6} \mathrm{yr}$ ), respectively.

be rewritten in terms of polar coordinates as follows:

$$
\theta-\theta^{\prime}=\left\{\begin{array}{ll}
0^{\circ} & \text { if } n>1 \\
180^{\circ} & \text { if } n<1
\end{array}\right\}, \quad \dot{\theta}<1,
$$

where $\theta^{\prime}$ is the longitude of Neptune, and $n$ is the mean motion of the test particle. In other words, the $(r, \dot{r})$ plane is equivalent to the $(X, \dot{X})$ plane with points plotted at every conjunction of the test particle with Neptune. The $(r, \dot{r}) \mathrm{s}-\mathrm{O}-\mathrm{s}$ is shown in Fig. 1(b).

Carrying this one step further by means of a canonical transformation to Delaunay variables $(M, J, \lambda, L)$, where $M, \lambda$ are the mean anomaly and mean longitude and $J$ $=a\left(1-\sqrt{1-e^{2}}\right), L=\sqrt{a\left(1-e^{2}\right)}$ [cf. Chap. XVII in Brouwer \& Clemence (1961) and Chap. 10 in Goldstein (1980)], this same section can also be seen in the $(M, J)$ plane. The $(M, J)$ s-o-s is shown in Fig. $1(\mathrm{c})$. It is evident from the latter figure that for Pluto-like resonance-locked orbits (i.e., for the particular value of $C$ chosen in this s-o-s) the mean anomaly at conjunctions with Neptune librates about $180^{\circ}$ and its maximum libration amplitude is $\sim 100^{\circ}$. Orbits with larger amplitudes are chaotic.

In addition to the maximum amplitude of $M$, the resonance libration region is bounded in $J$ as well. Now, if we note that (i) the Jacobi integral provides a relationship be- 

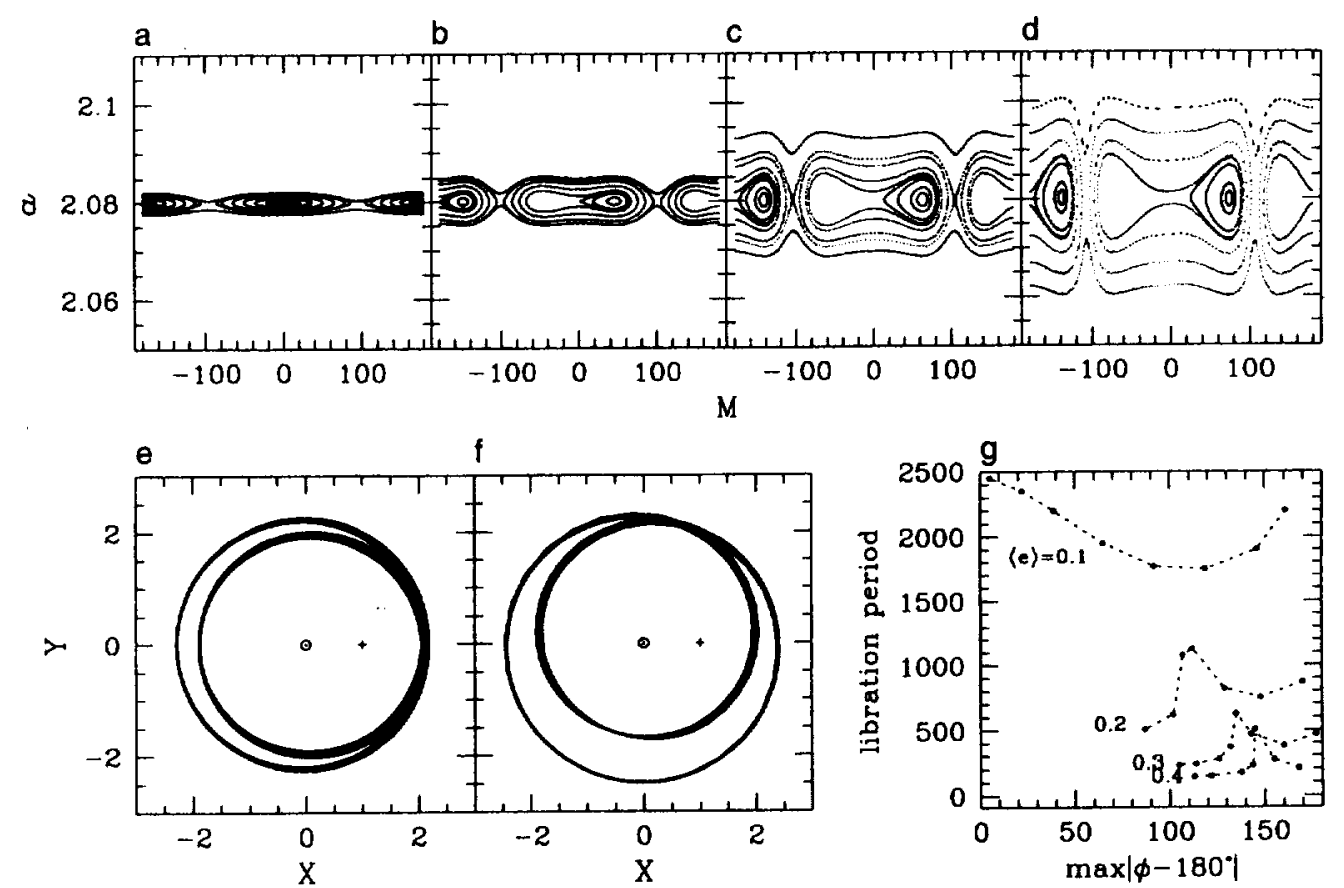

FIG. 3. The 3:1 Neptune resonance located at $a_{\text {res }}=2.08008$ (i.e., $62.6 \mathrm{AU}$ ). The phase space for orbits with (a) $C=3.3508,\langle e\rangle=0.1 ;(\mathrm{b}) C=3.3070,\langle e\rangle=0.2$; (c) $C=3.2324,\langle e\rangle=0.3 ;$ (d) $C=3.1244,\langle e\rangle=0.4$. The phase space is largely regular at this resonance. The width of the libration zone increases with $\langle e\rangle$. For $\langle e\rangle \geq 0.13$, the libration zone splits into two zones of asymmetric librations surtounded by a narrow zone of large-amplitude symmetric librations. Panel (e) shows a symmetrically librating orbit (with $e \sim 0.1$ ) in the rotating frame; panel (f) shows an asymmetrically librating orbit (with $e \sim 0.2$ ). The symbols $\odot$ and + indicate the fixed locations of the Sun and Neptune in the rotating frame. Panel ( $g$ ) shows the libration period (in units of Neptune's orbital period) as a function of the maximum excursion of the resonance angle $\phi$ from $180^{\circ}$. Note that the center of the asymmetric librators moves away from " $\phi=180^{\circ}$ " with increasing $\langle e\rangle$, as indicated by the beginning of the curves labeled by the different values of $\langle e\rangle$. The libration period increases with libration amplitude for the asymmetric librators, but decreases with amplitude for the symmetric (large-amplitude) librators; at the separatrix on the boundary between the symmetric and asymmetric librations, the libration period becomes indefnitely large (although this is not quite obvious due to the finite resolution in this figure).

tween $a$ and $e$ [see Eq. (4)], and (ii) for a particular orbital resonance defined by the ratio $(j+k): j$ of the orbital periods of the test particle and Neptune, the librating orbits all have a phase-averaged semimajor axis $\langle a\rangle=a_{\text {res }}$, then any s-o-s can be labeled uniquely either by the value of $C$ or, in the neighborhood of a particular orbital resonance, by the value of $\langle e\rangle$ (with the understanding that $\left.\langle a\rangle=a_{\text {res }}\right)$. For example, for the s-o-s in Fig. 1, we have $C=2.9798$; near the 3:2 resonance, $\langle a\rangle=1.3104$; therefore, from Eq. (4) we have $\langle e\rangle$ $=0.250$. Thus, the phase space structure in the neighborhood of a particular orbital resonance can be systematically studied in a set of surfaces-of-section where all of them have a common value of $\langle a\rangle$ but various values of $\langle e\rangle$ (hence different values of $C$ ). This allows a visualization of the phase space near an orbital resonance as a function of the mean orbital eccentricity.

Finally, in Fig. 1(d) I have plotted the values of $a$ against $M$ for the same points represented in Figs. 1(a)-1(c). Note that this is not a surface-of-section. One might call it a "pseudo-surface-of-section," for $a$ is not a canonical variable. However, the constancy of $C$ imposes a relationship between $a$ and $e$ [cf. Eq. (4)] which ensures that this plot looks very similar to the $(M, J)$ s-o-s in Fig. $1(b)$ and the stable libration regions and the chaotic regions are easily distinguished. The reason for choosing this noncanonical variable is that the resonance width $\Delta a$ of the stable libration zone can be readily determined from this figure. Therefore, the $(a, M)$ plane, the pseudo-surface-of-section, will be used in the next section for a systematic look at the properties of the phase space in the neighborhood of several Neptune resonances.

The libration time scale in the libration zone and the time scale for orbital instability in the chaotic zone are of particular interest for the dynamics of Kuiper Belt objects. These libration time scales have been determined for individual resonances and are also given in the next section. Typically, the timescale for orbital instability inside the chaotic zone surrounding a resonance is only a few times the libration period. However, close to the boundary between the libration zone and the chaotic zone, this time scale can become exceedingly large. In particular, for Pluto-like orbits with eccentricity $\sim 0.25$ in the $3: 2$ Neptune resonance, the timescale for instability in the chaotic zone (i.e., for amplitude of the resonance angle greater than $\left.\sim 140^{\circ}\right)$ is $Q\left(10^{5}\right)$ yr, but close to the edge of the libration zone this time scale increases by an order of magnitude or more. This is illustrated in Fig. 2.

\section{NEPTUNE RESONANCE ZONES}

In principle, there is an infinite number of orbital resonances in the three-body problem. However, we will see that only a handful warrant any detailed investigation.

Low-eccentricity orbits in the immediate neighborhood of a planet are unstable. There is a simple explanation for this 

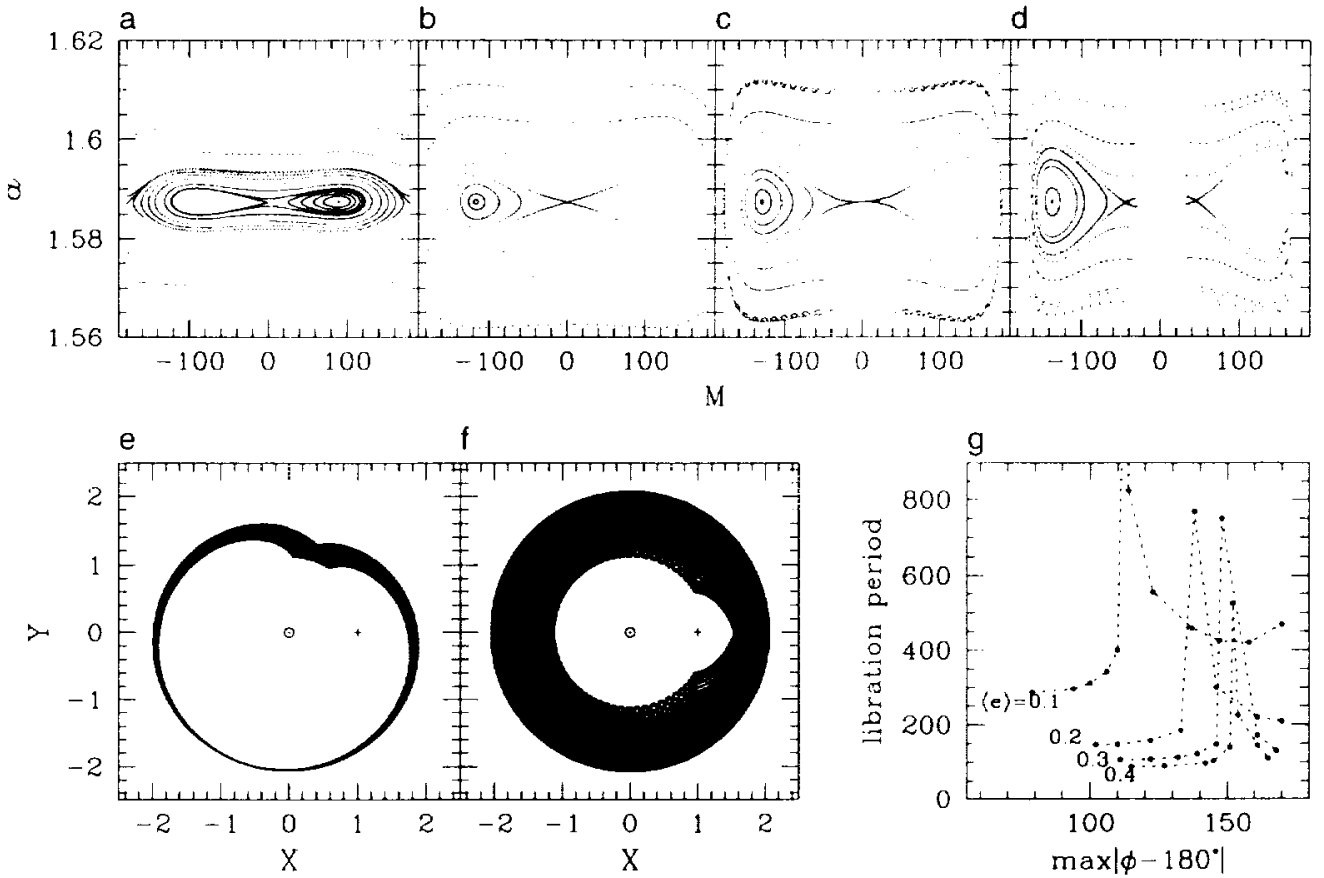

FIG. 4. The 2:1 Neptune resonance located at $a_{\text {res }}=1.5873$ (i.e., $47.8 \mathrm{AU}$ ). The phase space for orbits with (a) $C=3.1272,\langle e\rangle=0.1 ;(b) C=3.0989,\langle e\rangle=0.2$; (c) $C=3.0338,\langle e\rangle=0.3 ;$ (d) $C=2.9395,\langle e\rangle=0.4$. The phase space is largely regular at this resonance; a chaotic zone appears at large-amplitude librations for eccentricity exceeding $\sim 0.25$. The width of the libration zone increases with $\langle e\rangle$. For $\langle e\rangle \geqslant 0.04$, the libration zone splits into two zones of asymmetric librations surrounded by a narrow zone of large-amplitude symmetric librations. Panels (e) and (f) show a small-amplitude asymmetrically librating orbit and a large-amplitude symmetrically librating orbit (both with $e \approx 0.3$ ) in the rotating frame. (The motion of the test particle over a complete libration period is traced in these figures.) Note that in both types of orbits the perihelion occurs away from Neptune's longitude. Panel (g) shows the libration period (in units of Neptune's orbital period) as a function of the maximum excursion of the resonance angle $\phi$ from $180^{\circ}$. The center of the asymmetric librators is indicated by the beginning of the curves labeled by the different values of $\langle e\rangle$. The libration period increases with libration amplitude for the asymmetric librators, but decreases with amplitude for the symmetric (large-amplitude) librators; at the separatrix on the boundary between the symmetric and asymmetric librations, the libration period becomes indefinitely large

orbital instability that follows from Chirikov's (1979) "resonance overlap criterion": first-order $(j+1): j$ resonances with $(j+1) \geq 0.51 \mu^{-2 / 7}$ overlap completely (Wisdom 1980). [Numerical experiments by Duncan et al. (1989) show that the numerical coefficient on the right-hand side in this relation must be revised to 0.44]. Complete overlap of neighboring resonances results in the destabilization of all periodic and quasiperiodic orbits in the neighborhood of those resonances. In other words, circular test particle orbits are unstable in the immediate neighborhood $|a-1| \leqslant 1.5 \mu^{2 / 7}$ of a planet's orbit. For Neptune $\left(\mu=5.146 \times 10^{-5}\right)$, this criterion shows that all first-order resonances with $(j+1) \geqslant 8$ are completely overlapping, so that circular orbits beyond Neptune with orbital radii less than about $33 \mathrm{AU}$ are unstable.

Therefore, beyond the semimajor-axis range of about 33 $\mathrm{AU}$, at most only seven first order $(j+1): j$ resonances with $j=1,2, \ldots 7$ are isolated from each other (at least for low eccentricities). However, I have found numerically that loweccentricity orbits at the 8:7 and 7:6 Neptune resonances are also chaotic. This is not entirely surprising, for the resonance overlap criterion is quite approximate, and Wisdom's scaling law is strictly valid only in the asymptotic limit $j \gg 1$. Therefore, only five first-order $(j+1): j$ resonances with $j=1,2, \ldots 5$ are of potential significance for the long-term storage of Kuiper Belt objects.

Furthermore, since all second- and higher-order reso- nances in the first-order resonance overlap region are also destroyed, it follows that there are only seven second-order Neptune resonances of potential interest: $(j+2): j$ with $j=1,3,5, \ldots, 13$. Of these, 1 have found numerically that only three (3:1, 5:3, and 7:5) have resonance widths $\Delta a \geq 0.005$. Therefore, the phase space in the neighborhood of only these three second-order resonances will be discussed here.

Figures 3-10 show the properties of these first- and second-order Neptune resonances. They are arranged in order of decreasing mean heliocentric distance. Each figure has seven parts: the first four $[(a)-(d)]$ show the resonance zones in the pseudo-surfaces-of-section [plots of $(a, M)$ ] for $\langle e\rangle$ $=0.1,0.2,0.3$, and 0.4 , respectively; note that the vertical scale is the same in all these figures, so that the variations in the extent of the libration and chaotic zones with $\langle e\rangle$ and with the mean distance of the resonance from Neptune can be readily discerned. The next two panels $[(\mathrm{e})$ and (f)] show examples of librating, resonance-locked orbits in the rotating reference frame. The last one, panel $(\mathrm{g})$, shows the period of libration as a function of the mean orbital eccentricity and the amplitude of libration of the resonance angle $\phi$. A few words are in order regarding the latter variable.

For a $(j+k): j$ resonance, the resonance angle is defined as follows:

$$
\phi=(j+k) \lambda-j \lambda^{\prime}-k \varpi,
$$



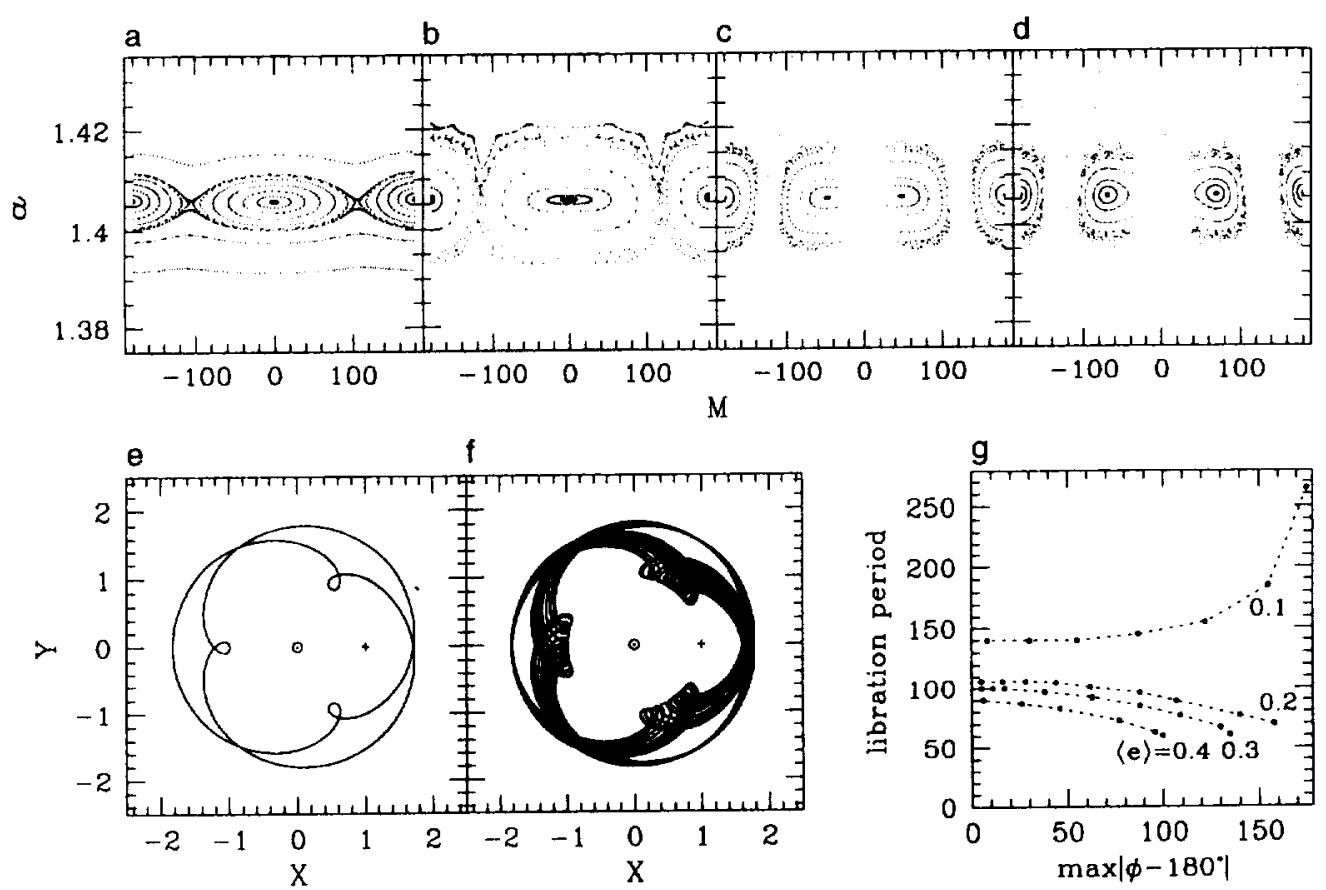

Fig. 5. The 5:3 Neptune resonance located at $a_{\text {res }}=1.4057$ (i.e., $42.3 \mathrm{AU}$ ). The phase space for orbits with (a) $C=3.0709,\langle e\rangle=0.1 ;(\mathrm{b}) C=3.0349,\langle e\rangle=0.2$; (c) $C=2.9739$, $\langle e\rangle=0.3$; (d) $C=2.8848,\langle e\rangle=0.4$. The width of the libration zone increases for $\langle e\rangle$ up to $\sim 0.2$, but then shrinks with increasing $\langle e\rangle$ as the larger-amplitude resonant orbits dissolve into the chaotic zone. Note that for $\langle e\rangle \geq 0.3$, the formerly period-2 exact resonant orbit in the surface-of-section becomes a period-3 orbit; this is merely a reflection of the fact that at large, Neptune-crossing eccentricities the perihelion loop results in two conjunctions with Neptune in rapid succession [cf. panels (e) and (f)]. Panels (e) and (f) show the periodic orbit at exact resonance, and an orbit librating with finite amplitude, respectively (both with $e \sim 0.3$ ) in the rotating frame. (The motion of the test particle over a complete libration period is traced in these figures.) Note that in both types of orbits the perihelion occurs away from Neptune's longitude. Panel (g) shows the libration period (in units of Neptune's orbital period) as a function of the libration amplitude of the resonance angle $\phi$. The libration period increases with libration amplitude only for small $\langle e\rangle$; it decreases with amplitude for larger $\langle e\rangle$.

where $\lambda$ and $\varpi$ are the mean longitude and longitude of perihelion of the test particle, and $\lambda^{\prime}$ is the mean longitude of Neptune. (Note that $j$ is a positive integer; $k$ is a negative integer for interior resonances, positive for exterior resonances; the latter are the only resonances of interest in this paper.) $\phi$ is the natural variable that arises in the perturbative analysis of orbital resonances, and it can be used to make a first approximation theory that models the resonant motion of the test particle with a single degree-of-freedom pendulum-like dynamical system (see, e.g., Malhotra 1994). For a test particle locked in a stable exterior resonance, $\phi$ librates about a mean value which is usually $180^{\circ}$. [There are two exceptions to this: (i) for sufficiently high eccentricity, librations of the resonance angle about $0^{\circ}$ are also possible; and (ii) the 2:1 and the 3:1 resonances allow for asymmetric librations where the center of libration of the resonance angle is displaced away from $180^{\circ}$. These are discussed further below.] At conjunctions with Neptune, the resonance angle is related to the mean anomaly: $\phi=j M+\mathcal{Q}(e)$. Therefore, the librations or chaotic variations of $M$ evident in the surfacesof-section are also reflected in the behavior of $\phi$.

The physical significance of the resonance angle can be seen by noting that when the test particle is at perihelion, $\lambda=\boldsymbol{\omega}$, so that

$$
\phi=j\left(\varpi-\lambda^{\prime}\right) \quad \text { (at perihelion). }
$$

Thus, the behavior of $\phi$ gives a direct measure of the longi- tude separation of Neptune from the test particle's perihelion. This is obviously a critical quantity, especially for higheccentricity Neptune-crossing orbits, as the stable libration of $\phi$ then ensures that the particle is protected from catastrophic close encounters with Neptune. The maximum excursion from the center of libration of $\phi$ then gives a measure of the smallest possible separation between Neptune and the test particle's perihelion in stable resonance-protected orbits.

The resonance libration period as a function of the amplitude of libration of $\phi$ is shown for various values of $\langle e\rangle$ in the last panel (g) of Figs. 3-10. These curves are rather counterintuitive. Recall that in the oft-used pendulum model for nonlinear resonances, the pendulum libration period increases monotonically with amplitude and becomes arbitrarily large close to the separatrix (i.e., the orbit that separates the oscillations from rotations of the pendulum). In contrast, in Figs. 3-10(g), we see that, except for the asymmetric librations at the 2:1 and 3:1 resonances, the libration period in general decreases with increasing libration amplitude. Furthermore, near a $k$ th order resonance, the leading order (in eccentricity) resonance term in the perturbing potential for the test particle is proportional to the $k$ th power of the eccentricity, hence the small-amplitude libration period might be expected to vary as $\sim e^{-k / 2}$; this analogy from the pendulum model does not hold either, as evident in Figs. 



FIG. 6. The 3:2 Neptune resonance located at $a_{\mathrm{res}}=1.3104$ (i.e., $\left.39.4 \mathrm{AU}\right)$. The phase space for orbits with (a) $C=3.0411,\langle e\rangle=0.1 ;(\mathrm{b}) C=3.0065 .\langle e\rangle=0.2$; (c) $C=2.9470,\langle e\rangle=0.3$; (d) $C=2.8616,\langle e\rangle=0.4$. The width of the main libration zone decreases with increasing $\langle e\rangle$, as the chaotic zone surrounding the libration region expands. Panel (e) shows an example of a stably librating orbit (with $e \approx 0.3$ ) in the rotating frame; note that this is an "aphelion librator": the test particle is near aphelion when passing Neptune's longitude, and its perihelion librates about $\pm 90^{\circ}$ away from Neptune. For $\langle e\rangle \geq 0.3$, a new libration zone appears in which the librating orbits have a perihelion near Neptune. This is indicated by the appearance of a chain of two libration islands (distinct from the main resonance zone) in panels (c) and (d); these are the "perihelion librators" [cf. panel (f)]: The new libration zone increases with increasing $\langle e\rangle$. An example of a perihelion librator is shown in panel (f); note that one of the two perihelion loops in each synodic period encompasses Neptune. Panel (g) shows the libration period (in units of Neptune's orbital period) of the aphelion librators as a function of the libration amplitude of the resonance angle $\phi$. The libration period generally decreases with libration amplitude, except for very small eccentricity $(e-0) .1)$, large-amplitude librators.

3-10(g). Thus, the standard nonlinear pendulum model should be used with caution for orbital resonances.

The general characteristics of the phase space near these first- and second-order Neptune resonances are summarized as follows. (Note that the 2:1 and the 3:1 resonances are exceptional in many respects, and are discussed later in detail.)

[cf. Figures 3-10(a)-(d)] In general, the phase space in the neighborhood of a resonance contains a zone of stably librating, resonance-locked orbits surrounded by a zone of chaotic orbits. The width of the resonance zone (including the stable as well as the chaotic regions) increases with $\langle e\rangle$; however, the width of the libration zonc, in general, shrinks as the resonance separatrix dissolves into a chaotic layer that increases in thickness with $\langle e\rangle$. The typical width of a Neptune resonance libration zone is $2 \Delta a \approx 0.02$, or about $0.6 \mathrm{AU}$.

[cf. Figures 3-10(e)] The periodic orbit at exact $(j+k): j$ resonance has a $j$-fold symmetry in the rotating frame: it makes $j$ perihelion passages during a synodic period. The perihelion (and aphelion) longitudes are spaced 36$)^{\circ} / j$. The quasi-periodic orbits librating about the exact resonance also exhibit $j$-fold symmetry in the rotating frame when traced over a complete libration period. For the stable librators (i.e.. resonance angle $\phi$ librating about $180^{\circ}$ ), the opposition with Neptune occurs near the test particle's aphelion; hence these may be called "aphelion librators."

[cf. Figures $6,7,9,10(f)]$ For sufficiently large $\langle e\rangle$, a new type of periodic (resonant) orbit is possible, where one of the $j$ perihelion passages during a synodic period occurs near Neptune. In fact, the perihelion loop encompasses Neptune. For this orbit and for the quasi-periodic orbits in its neighborhood, the resonance angle $\phi$ librates about $0^{\circ}$. These orbits may be called "perihelion librators." However, these perihelion librators are probably not of practical importance, as unmodeled perturbations are likely to destabilize them.

[cf. Figures $3-10(\mathrm{~g})]$ In general, the libration periods are smaller for resonances located closer to Neptune. and the libration periods decrease with libration amplitude. Typical small amplitude libration periods are $(1-2) \times 10^{4} \mathrm{yr}$.

The exceptional cases of the $2: 1$ and the $3: 1$ resonances are discussed below.

\subsection{The 2:I Neptune Resonance}

The synodic period of a test particle near the $2: 1$ resonance is approximately equal to its orbital period. Thus, conjunctions with Neplune occur every $\sim 330 \mathrm{yr}$. The propertics of the phase space near the 2:1 Neptune resonance are summarized as follows (cf. Fig. 4).

The resonance half width, $\Delta a$, increases from $\sim 0.001$ to $\sim 0.018$ as $\langle e\rangle$ increases from $\sim 0$ to 0.4 . The phase space at this resonance is largely regular; there is no discernible chaotic zone until the eccentricity exceeds $\sim 0.25$.

For $\langle e\rangle$ exceeding a critical value (which is $\sim 0.04$ for the 



FIG. 7. The 7:5 Neptune resonance located at $a_{\mathrm{re}}=1.2515$ (i.e., $37.7 \mathrm{AU}$ ). The phase space for orbits with (a) $C=3.0255,\langle e\rangle=0.1 ;(\mathrm{b}) C=2.9913,\langle e\rangle=0.2$; (c) $C=2.9334$, $\langle e\rangle=0.3$; (d) $C=2.8496,\langle e\rangle=0.4$. The width of the main libration zone (for aphelion librators) decreases rapidly with increasing $\langle e\rangle$, as the chaotic zone surrounding the libration region expands. In fact, for $\langle e\rangle \geqslant 0.3$ [cf. panel (d)] the aphelion libration zone has completely disappeared. A libration zone for perihelion librators appears at $e \approx 0.3$ and expands slightly with increasing $\langle e\rangle$. Panels $(e)$ and $(f)$ show examples of an aphelion librator with $e \approx 0.2$, and a perihelion librator with $e \approx 0.3$, respectively. Panel $(\mathrm{g})$ shows the libration period (in units of Neptune's orbital period) of aphelion librators as a function of the libration amplitude of the resonance angle $\phi$. The libration period generally decreases with libration amplitude, except for very small eccentricity $(e \leq 0.1)$, large-amplitude librators.

2:1 Neptune resonance), there are two types of librating orbits: (i) symmetric librators whose perihelia librate (with large amplitude) with a mean value $180^{\circ}$ away from Neptune, and (ii) asymmetric librators whose perihelia librate (with amplitude smaller than $\sim 90^{\circ}$ ) about a mean value which depends upon $\langle e\rangle$. (Surprisingly, the orbit with aphelion exactly at Neptune's longitude is unstable: it coincides with the separatrix that divides the phase space into the symmetric and asymmetric libration zones.) The existence of such asymmetric librations has been noted previously (e.g., Message 1958; Beauge 1994), but the work here provides the first quantitative analysis for the specific case of Neptune's resonances.

Figures 4(e) and 4(f) show examples of these two types of orbits. Note that for the asymmetric librators, there are two independent centers of libration, offset equally on either side of Neptune's longitude. Upon inspection of the behavior of the resonance angle $\phi$, it is found that these centers of perihelion libration (relative to Neptune) vary from $( \pm) 180^{\circ}$ for $\langle e\rangle \approx 0$ to $( \pm) 50^{\circ}$ for $\langle e\rangle \approx 0.4$.

The phase space for the symmetric librators shrinks rapidly with increasing $\langle e\rangle$, while that for the asymmetric librators increases slightly.

At small values of $\langle e\rangle$, there is no evidence for a chaotic zone near this resonance, but for $\langle e\rangle \geq 0.25$, the largest amplitude librators become chaotic. A thin chaotic zone also appears in the neighborhood of the separatrix that separates the asymmetric librations from the symmetric librations.

The eccentricity variations of the resonance-locked orbits have amplitude $\delta e \leq 0.01$. (Indeed, within the planar circular restricted three-body model, an initially circular test particle orbit at the $2: 1$ resonance has its eccentricity pumped up to only $\sim 0.03$.) The eccentricity variations on the chaotic orbits are generally of considerably larger magnitude.

Figure $4(\mathrm{~g})$ shows libration amplitude versus libration period. For the asymmetric (small amplitude) librators, the libration period decreases monotonically with $\langle e\rangle$ and increases with libration amplitude (reminiscent of the standard nonlinear pendulum). The libration period becomes indefinitely large at the separatrix between the asymmetric and symmetric librators. The small amplitude libration periods are several tens-of-thousands of years long.

\subsection{The 3:1 Neptune Resonance}

The synodic period of a test particle near the 3:1 resonance is half its orbital period. Thus, conjunctions with Neptune occur approximately every $248 \mathrm{yr}$. The properties of the phase space near the 3:1 Neptune resonance (cf. Fig. 3) are very similar to that near the 2:1 resonance; some specifics are noted below.

The resonance half width, $\Delta a$, increases from $\leqslant 0.001$ to $\sim 0.016$ as $\langle e\rangle$ increases from $\sim 0$ to 0.4 . The phase space at this resonance is regular; there is no discernible chaotic zone for this range of eccentricities.

For librating orbits, perihelion and aphelion occur at approximately the same longitude relative to Neptune. As in the $2: 1$ resonance, there are two types of librating orbits-the 

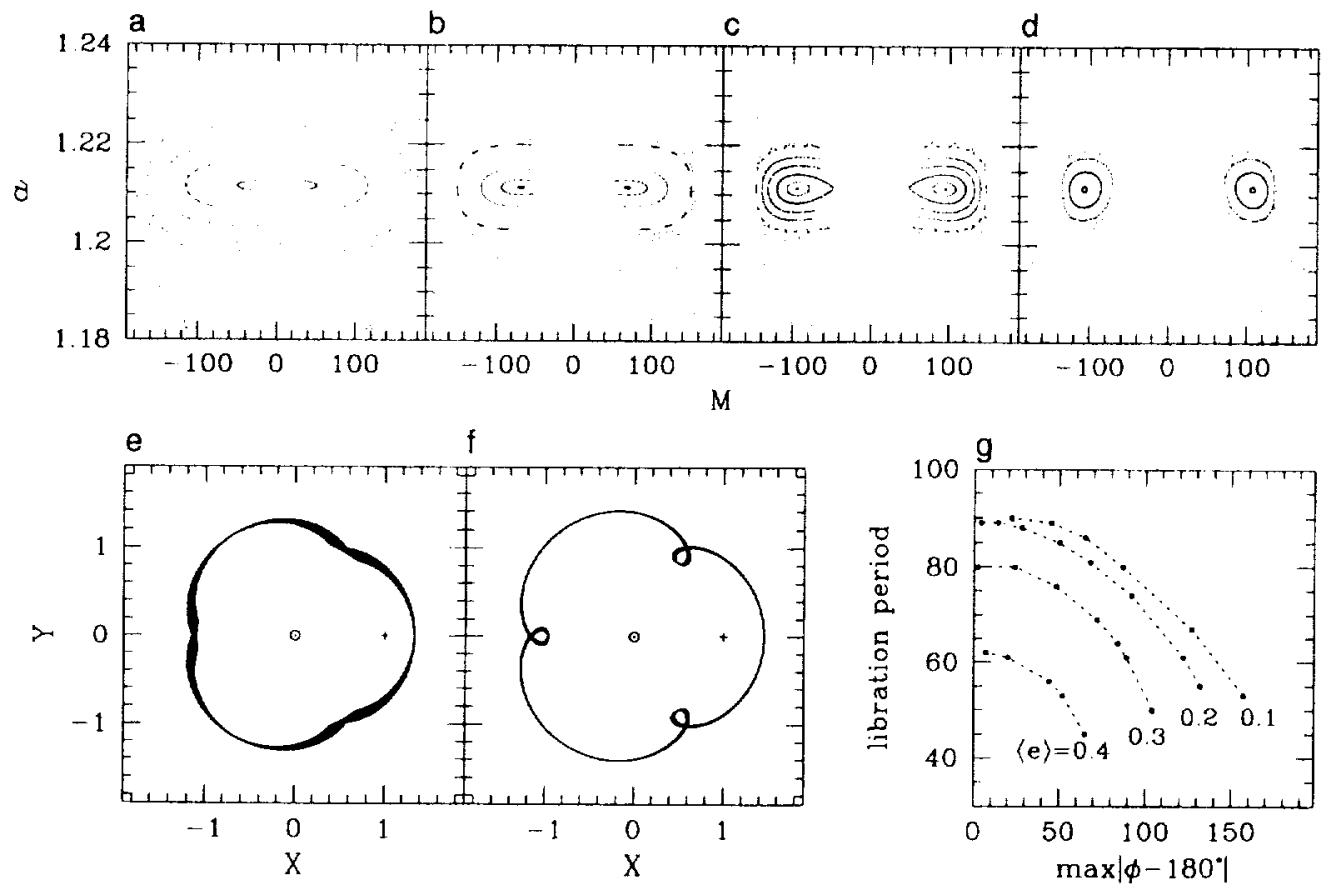

FiG. 8. The neighborhood of the Neptune $4: 3$ orbital resonance located at $a_{\mathrm{res}}=1.2114$ (i.e., $36.5 \mathrm{AU}$ ). The phase space for orbits with (a) $C=3.0158,\langle e\rangle=0.1$; (b) $C=2.9824$; $\langle e\rangle=0.2$; (c) $C=2.9253,\langle e\rangle=0.3$; (d) $C=2.8430,\langle e\rangle=0.4$. The libration zone shrinks rapidly with $\langle e\rangle$ as the larger amplitude librators dissolve into the chaotic zone. For $\langle e\rangle>0.175$, the formerly period-1 orbit corresponding to exact $4: 3$ resonance bifurcates into a period-2 orbit in the surface-of-section; this is merely a reflection of the fact that for a Neptune-crossing value of the eccentricity, the perihelion loop causes two conjunctions with Neptune in each synodic period. Panels (e) and (f) show examples of small amplitude librating orbit with $e \approx 0.1$ and $e \approx 0.2$, respectively. Panel (g) shows the period of libration as a function of the libration amplitude of the resonance angle. $\phi$ (for $\langle e\rangle=0.1,0.2,0.3$ and 0.4 ). The period as well as the maximum amplitude for stable librations decreases with increasing $\langle e\rangle$.

symmetric and asymmetric librators; however, the asymmetric librations appear only at $\langle e\rangle$ exceeding $\sim 0.13$.

The small amplitude libration periods are very long $(\sim 0.4$ Myr) for low eccentricity $e \leq 0.1$ orbits, but considerably shorter (30000-80000 yr) for higher eccentricities.

\section{SUMMARY AND DISCUSSION}

Numerical studies of the stability of low-eccentricity, lowinclination orbits of small objects in the trans-Neptunian Kuiper Belt subject to the gravitational perturbations of the giant planets have shown that the inner edge of the Kuiper Belt is at about $34 \mathrm{AU}$ heliocentric distance; beyond $34 \mathrm{AU}$ and up to about $42 \mathrm{AU}$ there are interspersed regions of stability and instability (Levison \& Duncan 1993; Holman \& Wisdom 1993). This structure bears a complex correlation with the locations of Neptune orbital resonances. Other studies (Malhotra 1993; Malhotra 1995) suggest that early in the history of the Solar system, the majority of Kuiper Belt objects were swept into eccentric orbits in narrow zones located at Neptune's orbital resonances. and that the regions inbetween the resonances would have been largely cleared of residual planetesimals. Recent and ongoing observational surveys of the outer Solar system indicate the presence of a large population of small bodies beyond Neptune (Jewitt \& Luu 1995). These are quite likely the source of short period comets (Duncan et al. 1988; Quinn et al. 1990). Furthermore, their orbital distribution is likely to hold clues to the formation and early dynamical evolution of the outer Solar system (Malhotra 1995). All of these considerations have motivated the present study.

Using the planar circular restricted three body model (with the Sun, Neptune, and a test particle), I have described in this paper the basic phase space structure in the neighborhood of Neptune's exterior orbital resonances. The details may be gleaned from Figs. 3-10. A succinct summary of these results is given in Fig. 11 which shows the locations and widths of the stable resonance libration zones. In general, these stable zones are bounded by chaotic layers of thickness that generally increases with eccentricity and decreases with distance from Neptune. (As the mean semimajor axis and eccentricity of chaotic orbits is not well defined, it is not possible to represent the chaotic layers in such a figure.)

The planar circular restricted three-body model is the simplest dynamical model for the orbital dynamics of small objects in the Kuiper Belt. Although this model may appear oversimplified, it provides a reasonable description and explanation for much of the dynamical behaviors found in the numerical studies mentioned above. ${ }^{1}$ For example, the location of the inner edge of this Belt at $\sim(33-34)$ AU is readily understood in terms of orbital instability induced by overlapping first-order Neptune resonances; the additional perturba-

\footnotetext{
'Those numerical studies assumed a realistic three-dimensional physical model in which test particles were perturbed by all the four giant planets, Jupiter, Saturn, Uranus, and Neptune; the planets' orbits were integrated self-consistently under their mutual gravitational perturbations.
} 

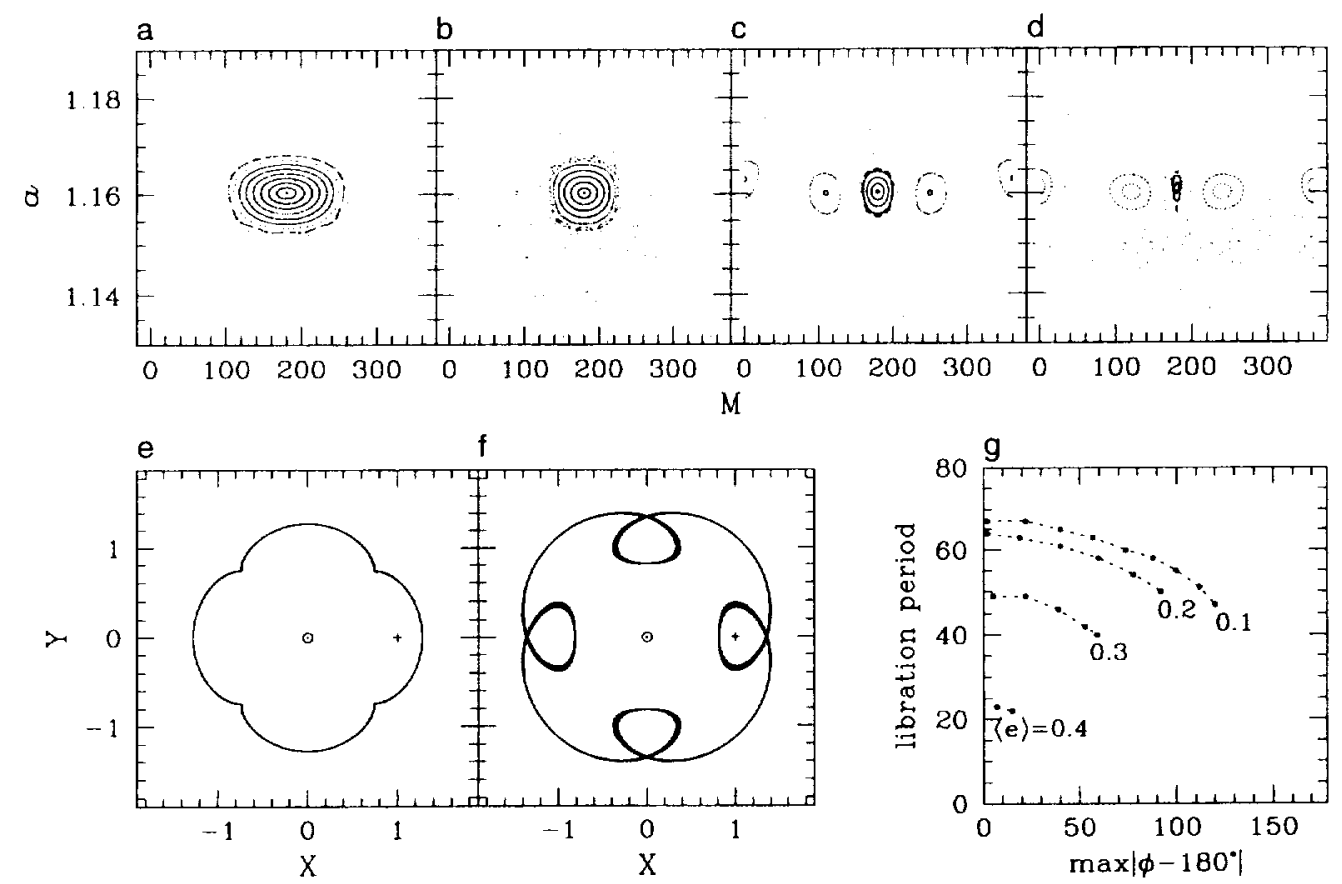

FiG. 9. The neighborhood of the Neptune 5:4 orbital resonance located at $a_{\mathrm{res}}=1.1604$. The phase space for orbits with (a) $C=3.0054$, $\langle e\rangle=0.1$; (b) $C=2.9727,\langle e\rangle=0.2 ;$ (c) $C=2.9170,\langle e\rangle=0.3$; (d) $C=2.8364,\langle e\rangle=0.4$. Notice that the main libration zone (for aphelion librators) shrinks rapidly with $\langle e\rangle$. A libration zone for perihelion librators appears for $\langle e\rangle>0.2$, as indicated by the appearance of a chain of two libration islands in (c) and (d); this zone increases with increasing $\langle e\rangle$. Panel (e) shows the stable periodic orbit with $e \approx 0.3$ at the $5: 4$ resonance (an aphelion librator) in the rotating frame coordinates; panel (f) shows an example of a perihelion librator with $e \approx 0.3$. Notice the fourfold symmetry in the rotating frame. Panel (g) shows the period of libration for the aphelion librators as a function of the libration amplitude of the resonance angle (for $\langle e\rangle=0.1,0.2,0.3$, and 0.4 )

tions due to Neptune's noncircular, inclined, and timevarying orbit and the perturbations of the other planets do not significantly change the location of this boundary.

The relatively isolated first- and second-order Neptune resonances beyond $34 \mathrm{AU}$ (in semimajor axis) provide narrow stable libration regions for the long-term storage of Kuiper Belt objects in eccentric (often Neptune-crossing) orbits. This paper provides first approximation estimates for the locations and widths of these regions, and for the dynamical properties of resonant orbits. It also provides a direct visualization, in two-dimensional surfaces-of-section, of the global phase space structure (i.e., the chaotic and stable regions) in the vicinity of orbital resonances. A characteristic of stable resonant orbits is the libration of the perihelion about a longitude well removed from Neptune's location. Typical libration periods are several tens-of-thousands of years. The libration zones are generally surrounded by narrow chaotic zones where orbits are unstable on timescales of a few libration periods, or $\sim 10^{5} \mathrm{yr}$. At the boundary between the stable resonance libration zone and the chaotic zone, the time scale for orbital instability may be exceedingly long. This paper also provides a quantitative analysis of libration periods and their dependence on libration amplitudes and orbital eccentricities at all the major exterior mean motion resonances of Neptune.

Of course, the planar circular restricted three-body model does not provide a complete picture. Indeed, I expect that it underestimates the extent of the chaotic zones and overestimates the sizes of the stable libration zones near Neptune resonances. But perhaps the most important missing element is the effect of secular resonances (see, e.g., Knezevic et al. 1991) on the long-term dynamics of Kuiper Belt objects. For example, in some regions, secular resonances produce significant inclination excitation; in such cases, the assumption of planarity becomes a poor approximation. [Pluto's orbit is a case in point (Nacozy \& Diehl 1978).] In order to discern these effects, one has to build much more elaborate analytical models or resort to extensive numerical integrations.

As this work was being completed, I became aware of two preprints (Morbidelli et al. 1995 and Duncan et al. 1995) on the same subject. These use different approaches to the problem: the present work has focussed on the phase space near mean motion resonances with Neptune only and gives results that are exact but for a highly simplified physical model; Morbidelli et al. (1995) discuss the dynamics near mean motion resonances as well as secular resonances using primarily approximate semi-analytic models; and Duncan et al. (1995) discuss the dynamics near mean motion resonances, secular resonances as well as nonresonant regions using a purely numerical approach. A comparison of the present work with these two preprints follows.

Morbidelli et al. (1995) describe semianalytic and numerical investigations that include the effects of all four giant planets on the dynamics of test particles in the Kuiper Belt. A comparison of their results for the locations and widths of mean motion resonances (see their Fig. 1) with the estimates obtained here (cf. Fig. 11) shows that their models significantly overestimate the sizes of the resonance libration 

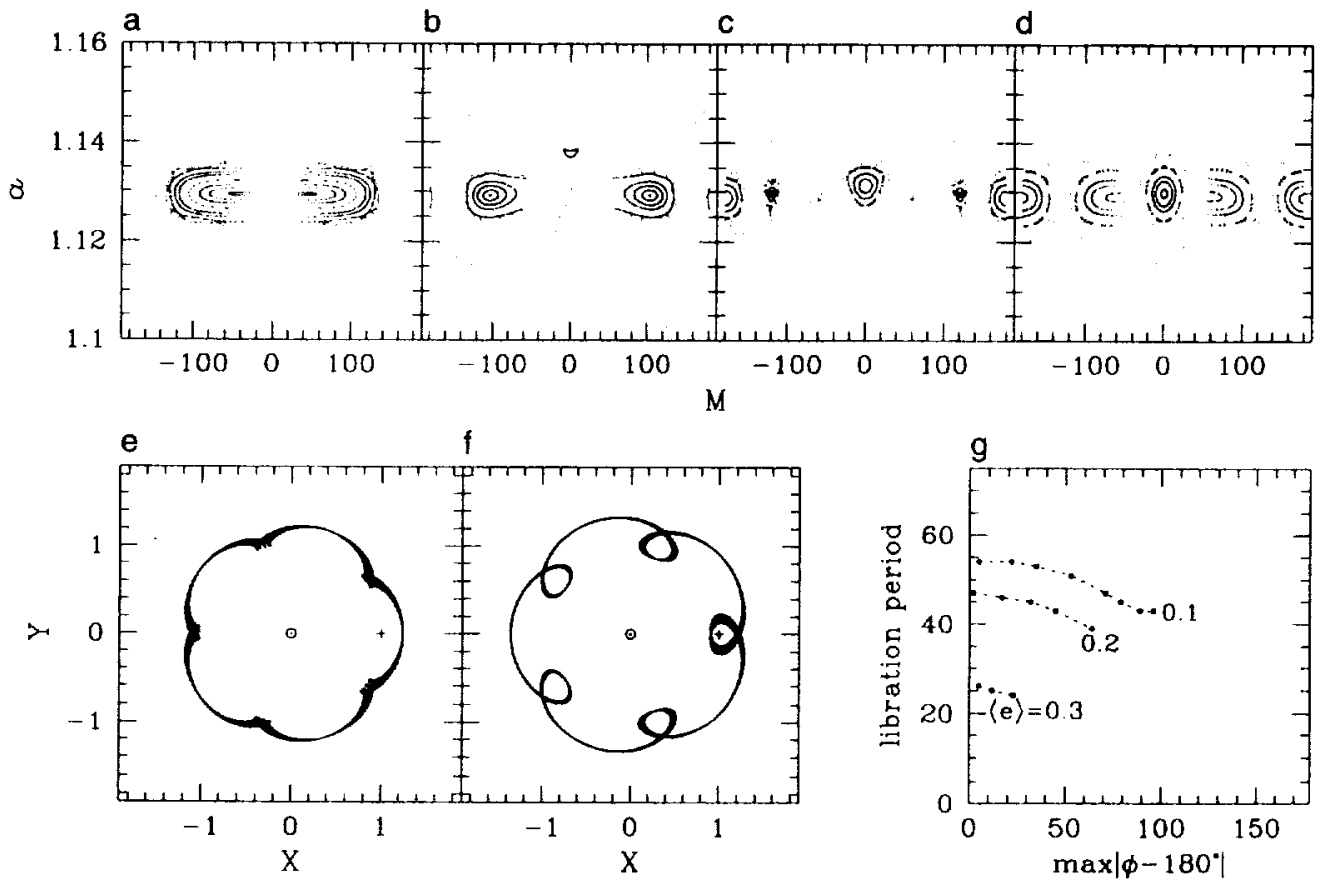

FIG. 10. The neighborhood of the Neptune $6: 5$ orbital resonance located at $a_{\text {res }}=1.1292$ (i.e., $34.0 \mathrm{AU}$ ). The phase space for orbits with (a) $C=3.0002$, $\langle e\rangle=0.1$; (b) $C=2.9680,\langle e\rangle=0.2$; (c) $C=2.9131,\langle e\rangle=0.3$; (d) $C=2.8334,\langle e\rangle=0.4$. Notice that the main libration zone (for aphelion librators) shrinks rapidly with $\langle e\rangle$; for $\langle e\rangle>0.1$ the center of this libration zone bifurcates into a period- 2 orbit; this is simply a reflection of the fact that the perihelion loop causes two conjunctions with Neptune in rapid succession during each synodic period. Also for $\langle e\rangle>0.1$, a new libration zone appears for perihelion librators [indicated by the appearance of a chain of two libration islands with centers at $M=0, \pi$ in (b)-(d)] whose extent increases with increasing $\langle e\rangle$. The increased complexity of this libration zone in (d) is a reflection of the fact that the perihelion loops for large-amplitude librations are so large that there are four conjunctions with Neptune during each synodic period. Panels (e) and (f) show examples an aphelion librator and of a perihelion librator with $e \approx 0.3$ at the $6: 5$ resonance. Notice the fivefold symmetry in the rotating frame. Panel $(\mathrm{g})$ shows the period of libration as a function of the libration amplitude of the resonance angle, $\phi$ (for $\langle e\rangle=0.1,0.2,0.3$ and 0.4 ) for the aphelion librators.

regions. Morbidelli et al.'s estimates of the resonance widths were obtained by averaging the planar, circular restricted problem of the four giant planets plus a test particle over fast variables, retaining only the dependence on the resonance angle. Thus, their analysis does not account for the overlapping secondary resonances at the edges of the mean motion

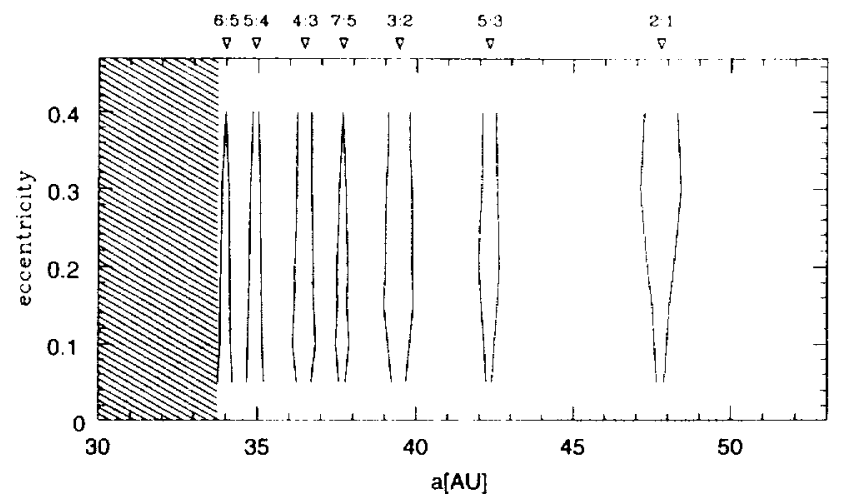

FIG. 11. The locations and widths of first- and second-order Neptune resonances in the Kuiper Belt, as determined by the planar, circular restricted three-body model. The shaded region on the extreme left indicates the chaotic zone of "resonance overlap" in the vicinity of Neptune's orbit. In the region above dotted line, orbits are Neptune-crossing; these orbits are dynamically short-lived (due to close encounters with Neptune), unless protected by orbital resonances. resonance libration zones that produce a chaotic layer and cause the libration regions to shrink rapidly with increasing eccentricity. This is the explanation for the differences between their Fig. I and Fig. 11 here. In addition, Morbidelli et al. find a singularity in the averaged Hamiltonian that causes a great increase in the resonance width near Neptunecrossing values of the eccentricity. However, this is an artifact, and does not appear in the unaveraged problem, as is obvious from the surfaces-of-section shown in the present work. The technique I used here does not make any averaging approximations, but uses the full unaveraged Hamiltonian for the circular planar restricted problem, albeit without the secular effects of Jupiter, Saturn, and Uranus. Morbidelli et al.'s models also show that secular effects are negligible near mean motion resonances except in the case of the 3:2 Neptune resonance. In the special case of the 3:2 resonance, as mentioned above, Knezevic et al. (1991) had previously shown the existence of the $\nu_{18}$ secular resonance embedded within the mean motion resonance; in addition, analysis of Pluto's orbit had also previously revealed the existence of another resonance characterized by the libration of the argument of perihelion (Nacozy \& Diehl 1978). Both of these resonances have the effect of exciting the inclination of the test particle. These resonances are not modeled in the present work, but Morbidelli et al. describe in some detail the location and widths of both these and other secular resonances. 
However, note that because most regions near secular resonances are regions of orbital instability, they are not likely to be of importance as reservoirs of Kuiper Belt objects although they may drive their transport to small heliocentric distances at the present epoch.

Duncan et al. (1995) have performed quite extensive numerical integrations of test particle orbits subject to the gravitational perturbations of the four giant planets. They describe their results in terms of the stability (over their integration time of 1-4 Byr) of orbits with particular initial semimajor axes, eccentricities, and inclinations. Their main conclusions are consistent with the results of the present work: namely, that particles with low initial inclination $\left(\sim 1^{\circ}\right)$ and initial perihelion distance less than $\sim 35 \mathrm{AU}$ are unstable on time scales short compared to the age of the Solar system, except that particles librating in low-order mean motion resonances with Neptune remain phase protected from close encounters with that planet. They have also analyzed the 3:2 Neptune resonance in more detail for the particular case with initial eccentricities of 0.2 and found three regimes: stable orbits deep in resonance with small libration amplitudes, $\leqslant 70^{\circ}$; an intermediate region with libration amplitudes in the range $70^{\circ}-130^{\circ}$ where the time scale for instability is $\sim 10^{9} \mathrm{yr}$; and highly unstable orbits for libration amplitudes exceeding $\sim 130^{\circ}$ with stability timescales of $\leqslant 10^{x} \mathrm{yr}$. Similar dynamics is found for all the Neptune resonances studied in this paper. In comparison, the present work has shown that the region of stability deep in resonance as well as the highly chaotic region at large amplitudes are both explained by the perturbations of Neptune alone (assumed to be on a circular orbit); the intermediate regime where the time scale for instability is $\sim 10^{9} \mathrm{yr}$ is very small in the restricted three-body model but evidently ex- pands greatly (at the expense of the stable libration zone) in the full $N$-body model for the outer Solar system. [In this context, we note that if Malhotra's (1995) theory for the orbital distribution of Kuiper Belt objects is correct, then this intermediate libration amplitude regime which allows a longterm leakage of resonant objects may be the primary source of the Jupiter-family short-period comets.] Duncan et al. also find that the resonance protection fails only at inclinations exceeding $\sim 25^{\circ}$. Thus, we conclude that the planar circular restricted three-body model used in the present work provides a fairly good description of these main trends. A clear advantage of this simple model is that its analysis is quite "inexpensive" compared to the months of CPU time expended in the numerical simulations by Duncan et al. Furthermore, the numerical integration of orbits for the age of the Solar system at sufficiently high resolution in the space of initial conditions remains beyond the reach of present-day computers, as acknowledged by Duncan et al. Thus, the global picture of the phase space structure obtained in the present work provides an inexpensive yet reasonably good baseline for understanding the dynamics in the transNeptunian Solar system, and is particularly useful near mean motion resonances.

This research was done while the author was a Staff Scientist at the Lunar and Planetary Institute which is operated by the Universities Space Research Association under Contract No. NASW-4574 with the National Aeronautics and Space Administration. Partial support for this work was also provided by NASA's Origins of Solar Systems Research Program under Grant No. NAGW-4474. This paper is Lunar and Planetary Institute Contribution No. 870.

\section{REFERENCES}

Bailey, M. E. 1983, Nature, 302, 399

Beauge, C. 1994, Calest. Mech. \& Dyn. Astron., 60, 225

Brouwer, D., \& Clemence, G. M. 1961, Celestial Mechanics (Academic Press, New York)

Chirikov, B. V. 1979, Phys. Rep., 52, 263

Duncan, M., Quinn, T., \& Tremaine, S. 1988, ApJ, 328, L69

Duncan, M., Quinn, T., \& Tremaine, S. 1989, Icarus, 82, 402

Duncan, M., Levison, H. F., \& Budd, S. M. 1995, preprint

Fernandez, J. A. 1980, MNRAS, 192, 481

Fernandez, J. A., \& Ip, W. H. 1983, Icarus, 54, 377

Goldstein, H. 1980, Classical Mechanics, 2nd ed. (Addison-Wesley, Reading. MA)

Hamid, S. E., Marsden, B. G., \& Whipple, F. L. 1968, AJ, 73, 727

Holman, M. J., \& Wisdom, J. 1993, AJ, 105, 1987

Jewitt, D. C.. \& Luu, J. X. 1993, Nature, 362, 730

Jewitt, D. C., \& Luu, J. X. 1995, AJ, 109, 1867

Knezevic, Z., Milani, A., Farinella, P., Froeschle, C. and Froeschle, C. 1991, Icarus, 93, 316
Kuiper, G. P. 1951, in Astrophysics: A Topical Symposium, edited by 1. A. Hynek (McGraw-Hill, New York), pp. 357-424

Levison, H. F., \& Duncan, M. J. 1993, ApJ, 406, L.35

Malhotra, R. 1993, Nature, 365, 819

Malhotra, R. 1994, Physica D. 77, 289 (Special issue on "Modeling the Forces of Nature")

Malhotra, R. 1995, AJ, 110, 420

Malhotra, R., \& Williams, J. G. 1994, in Pluto \& Charon, edited by D. Tholen and S. A. Stern (University of Arizona Press, Tucson) (in press) Marsden, B. G. 1995, MPEC 1995-L12; also MPECs 1995-EII, 1995-G04, 1995-G05, 1995-G13, 1995-K02, 1995-K04, 1995-L04

Message, P. J. 1958, AJ, 63, 443

Morbidelli, A., Thomas, F., \& Moons, M. 1995, preprint

Nacozy, P. E., \& Diehl, R. E. 1978, AJ, 83, 522

Quinn, T. R., Tremaine, S., \& Duncan, M. J. 1990, ApJ, 355, 667

Standish, E. M. 1993, AJ, 105, 2000

Whipple, F. L. 1964, Proc. Natl. Acad. Sci., 51, 711

Wisdom, J. 1980, AJ, 85, 1122 
$+$ 